\title{
Phytosocialogical and ethnomedicinal studies of sacred groves in konjikuppam village, cuddalore district, Tamil Nadu
}

\author{
J. Nithyadevi, R. Sivakumar \\ Department of Botany, Annamalai University, Aannamalai Nagar, Tamil Nadu, India \\ *E-mail address: herbnith@gmail.com
}

\begin{abstract}
The konjikuppam village lies on the state highway linking panruti (5 km south) and Neyveli (10 $\mathrm{km}$ north). The sacred grove of konjikuppam is also situated on the main read and is proximate to the village. A large bond of about 3 ha. Size lies behind the temple complex and cannel bringing strong water from the neighboring shallow ferralitic terrain runs into it. An extensive floristic survey of carried out in the sacred groves at monthly intervals between December 2011 and October 2012. Specimen flowering plants were collected and identified taxonomically with the help of different floras. Nine plots were established in three different disturbance areas within the sacred groves and it is divided into three site I. Disturbances, II. Moderately disturbance III. Undisturbance. Present study revealed that a total number of 110 plants belonging to 96 genera and 45 families were recorded from three sites (I. Disturbed, II. Moderately Disturbed, III. Undisturbed) of konjikuppam sacred groves Cuddalore district. The present study revealed that more number of species found in undisturbed site III and least number and density in disturbed site I. a total of 24 plants used in herbal preparations. The local health traditions provide immediate and cheaper remedy or relief to the poor and down trodden inhabiting the villages. The devastation of species diversity in the study area there is an urgent need for regeneration of the species for conservation of species and biodiversity.
\end{abstract}

Keywords: Phytosocialogical; Sacred groves; Ethnomedicine; Biodiversity

\section{INTRODUCTION}

Biodiversity studies have been carried out in different plant communities from plains to the hilltops. There are various plant communities that exist as sacred groves distributed throughout India, which are seen as relict of the ancient vegetation, generally rich in plant diversity (Harikrishnan Nair et al., 1997) the protected refugia of the natural ecosystem in a given region have exited as sacred groves in many societies all over the world. The groves are small patches of vegetation types that were traditionally protected and managed by the local communities, through a wide range of management practices (Gadgil and Vartak, 1976).

In India 13,720 sacred groves have been identified in various parts of India the sacred groves are the representative of climax vegetation and exhibit the diversity of species such as trees, climbers, epiphytes and other shade loving herbs (Bhandary and Chandrasekar, 2003).many people have described sacred groves in different ways. However, thare is an evident fact that wherever sacred groves exited, because of indigenous traditional socities has spiritual relationship with the exiting physical environment. The role of sacred groves in the conservation of biodiversity has long been recognized (Gadgil and Vartak, 1976; Osambi, 1962; Haridasan and Rao, 1985; Khan et al, 199; Ramanujam and Kadamban, 2001; 
Ramanujam and Pravin Kumar Cyril, 2003; Nadanakunjidam and Kamashi, 2003; Nadanakunjidam and Abirami, 2005; Nadanakunjidam, 2006) Vartak and Gadgil have traced this historical link of the sacred groves to the agricultural, hunting and gathering societies (Vartak et al., 1973)

Folk medical practitioners are called Nattu Vaidhyans in South India, or Vaidu or Amchi in others. A social dimension also has emerged. Nowadays, people look forward to a long term cure without side effects rather than a short term relief accompanied by lots of illeffects. Traditional medical systems may be a slow science but the west is gradually turning towards the natural systems of cure and care. The present study enlists the species composition of three sacred groves and importance of traditional medicinal plants in Konjikuppam Village, Cuddalore District.

\section{MATERIAL AND METHODS}

Konjikuppam village lies on the state highway linking Panruti (5 km south) and Neyveli (10 km North). There are 125 households with a population of ca. 500 people. The sacred grove of Konjikuppam is also situated on the main road and is proximate to the village. It measures 4.5 ha. The terrain is gently undulating and the temple complex is situated on the south-east corner down the slope. A large bond of about 3 ha.

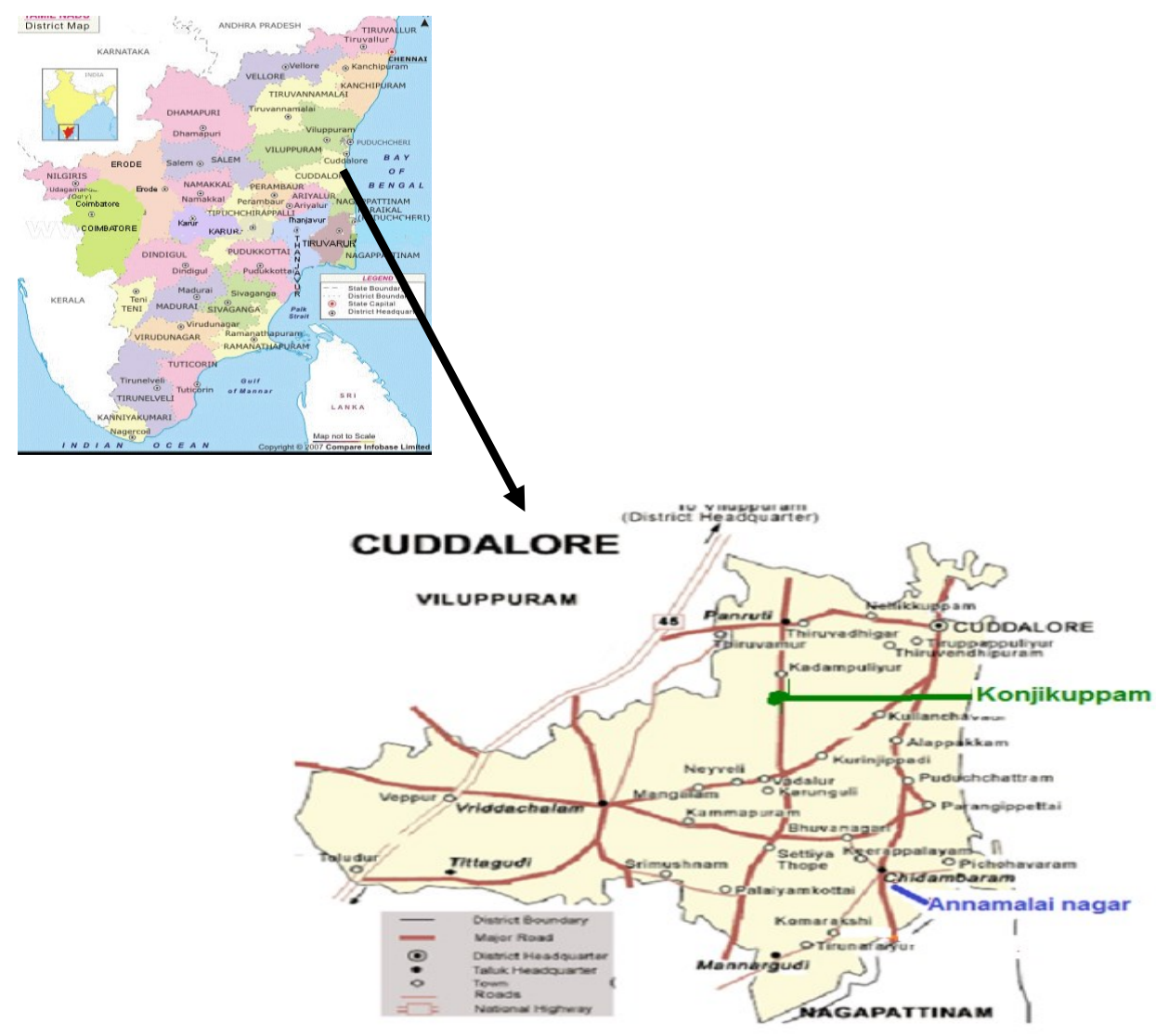

An extensive floristic survey was carried out in the sacred groves at monthly intervals between December 2011 and October 2012. The sacred groves and it is divided into three sites 1. Disturbed, 2. Moderately disturbed, 3. Undisturbed. In each forest type three $50 \mathrm{~m} \mathrm{x}$ $50 \mathrm{~m}$ plots were randomly setup. Each plot was subdivided into four $25 \mathrm{~m} \times 25 \mathrm{~m}$ quadrate for easy sampling. 


\section{1. Vegetation analysis}

The vegetation data were analyzed for relative frequency, relative density, and relative dominance. The sum of relative frequency, relative density and relative dominance represented the Importance Value Index (IVI) for various species (Curtis, 1959).

Frequency: Frequency is the number of sampling units (as \%) which a particular species occurs. Thus frequency of each species calculated as follows:

$$
\text { Frequency }=\frac{\text { Number of smapling units in which the species occured }}{\text { Total number of sampling units studied }} \times 100
$$

Density: Density represents the numerical strength of a species in the community. The number of individuals of the species in any unit area is its density. Density gives an idea of degree of competition. It is calculated as follows:

$$
\text { Density }=\frac{\text { Number of individuals of the species in all sampling units }}{\text { Number of sampling units studied }}
$$

The value thus obtained is then expressed as number of individuals per unit area.

Abundance: This is the number of individuals of any species per sampling unit of occurrence. It is calculated as follows:

$$
\text { Abundance }=\frac{\text { Number of individuals of the species in all sampling units }}{\text { Number of sampling units in which the species occured }}
$$
formula:

Since most of the stems are cylindrical, the basal area was calculated by using the Basal area $=\pi \mathrm{r} 2$

Where,

$\pi=3.14$ and ' $r$ ' is the radius of the stem at the point of emergence.

Relative frequency: The dispersion of species in relation to that of all the species is termed as relative frequency of a species.

$$
\text { Relative frequency }=\frac{\text { Frequency of the species in a quadrat }}{\text { Sum of the frequencies for all species in the same quadrat }} \times 100
$$

Relative density: The proportion of density of a species to that of stand as a whole is referred to as relative density.

$$
\text { Relative density }=\frac{\text { Number of individuals of a species }}{\text { Number of individuals of all species }} \times 100
$$




\section{Relative abundance:}

$$
\text { Relative abundance }=\frac{\text { Abundance of a species }}{\text { Total abundance of all species }} \times 100
$$

Importance value Index (IVI) is the sum of quantities of relative frequency, relative density and relative dominance expressed per 300 .

$$
\text { Relative Importance value index }=\frac{\text { IVI of the species }}{3}
$$

Species diversity: Species diversity is the ratio between the number of species and importance value or number or biomass or productivity and it was calculated using the formula given by Margalef (1968);

$$
\text { Where, } \begin{aligned}
\mathrm{H}^{\prime} & =- \text { ? }(\mathrm{ni} / \mathrm{N}) \ln (\mathrm{ni} / \mathrm{N}) \\
\mathrm{Ni} & =\text { importance value index of species } \mathrm{i} ; \\
\mathrm{N} & =\text { importance value index of the community. }
\end{aligned}
$$

Dominance index: the dominants are the plants by virtue of their abundance, growth performance and dry matter production and become conspicuous in a community concentration of dominance (C) was calculated by Simpson's index (Simpson, 1949).

$$
\mathrm{C}=?(\mathrm{ni} / \mathrm{N})^{2}
$$

Where, $\mathrm{C}=$ index of dominance $n i$ and $N$ being same as in the Shannon index of general diversity.

Species richness: Species richness is an indicator of the relative wealth of species in a community (Peet, 1974) and it can be represented by the total number of species in a given community or the number of species per unit area. Species richness was calculated by following formula by (Menhinick, 1964).

$$
S R=\frac{S}{\log 10 S}
$$

Species evenness: species evenness represents distribution of individuals among the species. It sometimes defined as the ratio of observed diversity to maximum diversity (Margalef, 1958). The evenness of equitability was calculated by the formula suggested by the Pielou (1966) as

$$
\mathbf{S R}=\frac{\mathbf{S}}{\sqrt{n}}
$$

Where $\mathrm{S}=$ number of species, $\mathrm{n}=$ number of individuals

Index of similarity: The interspecific association can be evaluated by association index and also by calculating index of similarity. The index of similarity is utilized to compare two coexisting groups was calculated following Odum (1971) as 


\section{Index of similarity $=\frac{2 \times \text { Numbers of common species }}{\text { Total number of species in both association }} \times 100$}

$$
\mathrm{S}=2 \mathrm{c} / \mathrm{a}+\mathrm{b}
$$

Where $\mathrm{a}=$ number of species in the sample A;

$b=$ number of species in the sample $B$ and

$\mathrm{c}=$ number of species common to both samples.

\section{2. Ethnomedicinal studies}

The work was carried out adopting the methodology of Jain (1989 and 1995). The surveys were spread across seasons so as to get maximum information and also to cross check the information provided by the local informants during the earlier visits. Structured questionnaires, interviews, and participatory observations were used to illicit information from the resource persons using standard methods [Martin, 1995].

\section{RESULT AND DISCUSSION}

Floristic analyses were carried out in disturbed moderately disturbed, and undisturbed of study area. Through there a grove is located in the main road and is proximate to the village, a major portion of the groves remains undisturbed. Theses groves inhabit 110 plant species belonging to 96 genera and 48 families (Table 1).

Tree species richness varied according to the disturbance gradient in the different stands. Consolidated data of phytosociological studies are given in (Table- 5). Tree species richness (number of species) was higher in site III (26 species) followed by Site II (17 species) and Site I ( 9 species). Site III stand was found to have greater density ( $83 \mathrm{no} / \mathrm{ha})$, diversity index $(0.254)$, basal area $\left(13.311 \mathrm{~m}^{2} / \mathrm{ha}\right)$ and species richness (1.566). However, dominance index (0.267) and evenness index (2.154) were recorded in site I stand. Number of species (species), basal area $\left(4.565 \mathrm{~m}^{2} / \mathrm{ha}\right)$, density (19 no/ha), species richness $(0.863)$ and diversity index (1.103) were found to be least in site I stand. The highest tree species diversity was recorded in the sites III, II stands and lowest in site I stand.

Dominance calculated as the IVI of different species varied greatly indifferent stands. The IVI of trees in the study area is given in (Table - 4) Greater number of tree species (26) was recorded in site I stand followed by site II stand (17), whereas least number of species (9) was seen in site I stand. Albizia amara in site I, II and III stands and Mimusops elengi and Madhuca longifolia in site I stand and Lepisanthes tetraphylla and Tricalysia apiocarpa in site III were found as the dominant tree species, whereas Ficus racemosa in site I stand were the least dominant in terms of IVI.

\section{1. Seedlings}

In seedling population higher in number of species (22), species diversity index (2.209) was observed in site III stand. Evenness index (2.247) in site II stand and dominance index (0.324) in site I stand was found to be greater. However, density (630/ha) basal area $\left(1.301 \mathrm{~m}^{2} / \mathrm{ha}\right)$, diversity index (1.048), and evenness index (2.021) in site I stand were least. 
(Table-5)Greater number of seedling species (22) was recorded in site III stand was followed by site II and I. Seedlings of Albizia amara, Chloroxylon swietenia were seen in three sites whereas the seedlings of Diospyros montana, Tricalysia apiocarpa, Premna tomentosa were found in single sites.

\section{2. Saplings}

Among the sapling populations number of species (11), basal area $\left(8.050 \mathrm{~m}^{2} / \mathrm{ha}\right)$, diversity index (2.150) and species richness (2.157) in site III stand were found to be greater. However, the density (190), and evenness index (2.183) in site II stand and dominance index (0.430) in site I stand were greater. Number of species (2) in site I stand was the least. Density $(120 / \mathrm{ha})$, basal area $\left(2.356 \mathrm{~m}^{2} / \mathrm{ha}\right)$ and species richness $(0.800)$, diversity index $(0.958)$ and evenness index (2.007) in site I stand, and dominance index (0.104) in site III, were the least (Table -5). Compared with IVI, Lepisanthes tetraphylla shows greater (51.42) and least in Ficus racemosa $(1.75)$

\section{3. Shrubs}

Among the shrub populations number of species (18), diversity index (2.307), species richness (1.336), eveness index (2.250) in site III stand were greater. However, the dominance index $(0.159)$ in site I stand and basal area $\left.\left(2.213 \mathrm{~m}^{2} / \mathrm{ha}\right)\right)$ were found to be higher. In site I stand basal area $(1.057 \mathrm{~m} 2 / \mathrm{ha})$ were found to be least (Table-5). Higher number of shrubs species (18) was recorded in site III and followed by I and II stands. Least number of shrubs species (6) was found in site I stand. Cassia auriculata Clausena dentata, Glycosmis mauritiana, Memecylon umbellatum, Mimosa intsia were found in all study sites, whereas Catunaregam spinosa, Grewia hirsuta, Opuntia dilleni and Tragia plukeneti were found in single sites. Compared with IVI, Glycosmis mauritiana shows greater (52.32) and least in Cissus quadrangularis (0.38) (Table-2).

\section{4. Herbs}

Number of species (43), diversity index (2.033), eveness index (2.104) and species richness (1.897) in site III stand were greater. However the density (140/ha), basal area $\left(1.314 \mathrm{~m}^{2} / \mathrm{ha}\right)$ in site II and dominance index $(0.141)$ in site I were greater. Least number of species (12), density (4250 ha, basal area $\left(0.063 \mathrm{~m}^{2} /\right.$ ha), diversity index $(1.637)$ and evenness index (1.839) in site I and species richness (0.913) and dominance index (0.127) in site III stands were recorded least (Table-5). The density of Acanthaceae, Rutaceae, and Capparidaceae in site III stand were higher, whereas the density of Cactaceae and Lythraceae in site I stand, Nyctaginaceae in site II stand and Solanaceae and Violaceae in site III stand were having least density. Compared with IVI, Ageratum conyzoides shows greater (16.65) and least in Vernonia cinerea (0.55) (Table-3). 
Table 1. List of species encountered at three sites of konjikuppam sacred groves.

\begin{tabular}{|c|c|c|}
\hline S.No & FAMILY & BOTANICAL NAME \\
\hline 1. & Boraginaceae & Cormona retusa (Vahl) Masam \\
\hline 2. & Cactaceae & Opuntia dillenii (Ker-Gawler) Haw. \\
\hline 3. & Caesalpiniaceae & Senna auriculata (L.) Roxb. \\
\hline 4. & Capparidaceae & Cadaba fruticosa (L.) Druce \\
\hline 5 & Capparidaceae & Capparis divaricata Lam. \\
\hline 6 & Capparidaceae & Capparis rotundifolia Rottl. \\
\hline 7 & Capparidaceae & Capparis sepiaria Linn. \\
\hline 8 & Euphorbiaceae & Jatropha gossypifolia L. \\
\hline 9 & Euphorbiaceae & Tragia plukeneti R.Smith \\
\hline 10 & Flacourtiaceae & Flacourtia indica (Burm.f) Merr \\
\hline 11 & Linaceae & Hugonia mystax L. \\
\hline 12 & Melostomaceae & Memecylon umbellatum Burm.f. \\
\hline 13 & Mimosaceae & Mimosa caesia $\mathrm{L}$. \\
\hline 14 & Ochnaceae & Ochna obtusata DC. \\
\hline 15 & Rhamnaceae & Zizyphus oenoplia (L.) Merr. \\
\hline 16 & Rubiaceae & Canthium coromandelianum (Burm.f.) Alston \\
\hline 17 & Rubiaceae & Catunaregam spinosa (Thunb.) Triveng \\
\hline 18 & Rubiaceae & Ixora pavetta Andrews \\
\hline 19 & Rubiaceae & Tarenna asiatica (L.) Kuntze ex K. Schum \\
\hline 20 & Rutaceae & Clausena dentata (Willd.) M. Roem \\
\hline 21 & Rutaceae & Glycosmis mauritiana (Lam.) Yuich.Tanaka \\
\hline 22 & Sapindaceae & Dodonaea angustifolia L.f. \\
\hline 23 & Tiliaceae & Grewia hirsuta Vahl \\
\hline 24 & Acanthaceae & Andrographis paniculata (Burm.f) Wall.ex Nees \\
\hline 25 & Acanthaceae & Asystasia gangetica (L.) T.Ander \\
\hline 26 & Acanthaceae & Barleria cuspidata Heyne ex Nees \\
\hline 27 & Acanthaceae & Barleria prionitis L. \\
\hline 28 & Acanthaceae & Blepharis molluginifolia Pers. \\
\hline 29 & Acanthaceae & Ecbolium ligustrinum (Vahl) Volleson \\
\hline 30 & Acanthaceae & Elytraria acaulis (L.f.) Lindau \\
\hline 31 & Acanthaceae & Hygrophila auriculata (Schum.) Heine \\
\hline 32 & Acanthaceae & Lepidagathis cristata Nees \\
\hline 33 & Amaranthaceae & Achyranthes aspera L. \\
\hline 34 & Amaranthaceae & Allmania nodiflora (L.) R.Br. ex Wight. \\
\hline 35 & Amaranthaceae & Alternanthera sessilis (L.) R.Br. ex DC. \\
\hline 36 & Asteraceae & Acanthospermum hispidum DC. \\
\hline 36 & Asteraceae & Ageratum conyzoides L. \\
\hline 37 & Asteraceae & Eclipta prostrata (L.) L. \\
\hline 38 & Asteraceae & Sonchus oleraceus L. \\
\hline 39 & Asteraceae & Sphaeranthus indicus L. \\
\hline 40 & Asteraceae & Vernonia cinerea L. \\
\hline 41 & Boraginaceae & Coldenia procumbens L. \\
\hline 42 & Boraginaceae & Heliotropium indicum L. \\
\hline 43 & Cactaceae & Cereus trigonus Lem. \\
\hline 44 & Caesalpiniaceae & Senna tora (L.) Roxb. \\
\hline 45 & Capparidaceae & Cleome aspera Koenig Ex DC. \\
\hline 46 & Capparidaceae & Cleome monophylla L. \\
\hline 47 & Capparidaceae & Cleome viscosa L. \\
\hline 48 & Commelinaceae & Commelina bengalensis L. \\
\hline 49 & Commelinaceae & Commelina erecta $\mathrm{L}$. \\
\hline 50 & Fabaceae & Alysicarpus monilifer (L.) DC. \\
\hline 51 & Fabaceae & Desmodium triflorum (L.) DC. \\
\hline
\end{tabular}




\begin{tabular}{|c|c|c|}
\hline 52 & Fabaceae & Indigofera aspalathoides Vahl. ex DC. \\
\hline 53 & Fabaceae & Zornia triphylla (L.) Pers. \\
\hline 54 & Lamiaceae & Leucas aspera (Willd.) Link \\
\hline 55 & Lamiaceae & Ocimum canum Sims \\
\hline 56 & Lythraceae & Ammania baccifera $\mathrm{L}$. \\
\hline 57 & Malvaceae & Sida cordifolia L. \\
\hline 58 & Molluginaceae & Mollugo nudicaulis Lam. \\
\hline 59 & Nyctaginaceae & Boerhaavia diffusa $\mathrm{L}$. \\
\hline 60 & Poaceae & Cenchrus ciliaris L. \\
\hline 61 & Poaceae & Chloris barbata Sw. \\
\hline 62 & Poaceae & Chrysopogon aciculatus (Retz.) Trin. \\
\hline 63 & Poaceae & Cynodon dactylon (L.) Pers. \\
\hline 64 & Poaceae & Dactyloctenium aegyptium (L.) Willd. \\
\hline 65 & Poaceae & Dichanthium annulatum (Forssk.) Stapf. \\
\hline 66 & Poaceae & Diplachne fusca (L.) Beauv. ex Roem. et Sch. \\
\hline 67 & Poaceae & Perotis indica (L.) Kuntze \\
\hline 68 & Rubiaceae & Oldenlandia herbacea (L.) Roxb. \\
\hline 69 & Rubiaceae & Oldenlandia umbellata L. \\
\hline 70 & Scrophulariaceae & Scoparia dulcis L. \\
\hline 71 & Solanaceae & Physalis minima L. \\
\hline 72 & Solanaceae & Solanum nigrum L. \\
\hline 73 & Tiliaceae & Corchorus aestuans L. \\
\hline 74 & Tiliaceae & Triumfetta rhomboidea Jacq. \\
\hline 75 & Verbenaceae & Phyla nodiflora (L.) Greene \\
\hline 76 & Verbenaceae & Stachytarpheta jamaicensis (L.) Vahl \\
\hline 77 & Violaceae & Hybanthus enneaspermus (L.) F.Muell. \\
\hline 78 & Zygophyllaceae & Tribulus terrestris L. \\
\hline 79 & Alangiaceae & Alangium salvifolium (L.f.) Wang \\
\hline 80 & Anacardiaceae & Lannea coromandelica (Houtt.) Merr. \\
\hline 81 & Boraginaceae & Cordia monoica Roxb. \\
\hline 82 & Boraginaceae & Cordia obliqua Willd. \\
\hline 83 & Caesalpiniaceae & Cassia fistula L. \\
\hline 84 & Celastraceae & Cassine glauca (Rottb.) Kuntze \\
\hline 85 & Ebenaceae & Diospyros melonoxylon Roxb. \\
\hline 86 & Ebenaceae & Diospyros montana Roxb. \\
\hline 87 & Meliaceae & Azadirachta indica A.Juss \\
\hline 88 & Meliaceae & Walsura trifoliata ( A.Juss) Harms \\
\hline 89 & Mimosaceae & Acacia nilotica ssp.indica (Benth.) Brenan \\
\hline 90 & Mimosaceae & Acacia auriculiformis A.Cunn. ex.Benth. \\
\hline 91 & Mimosaceae & Albizia amara (Roxb.) Boivin \\
\hline 92 & Mimosaceae & Albizia lebbeck (L.) Benth. \\
\hline 93 & Mimosaceae & Dichrostachys cinerea (L.) Wt. \& Arn. \\
\hline 94 & Mimosaceae & Mimosa intsia L. \\
\hline 95 & Moraceae & Ficus amplissima Smith \\
\hline 96 & Moraceae & Ficus racemosa $\mathrm{L}$. \\
\hline 97 & Moraceae & Ficus religiosa L. \\
\hline 98 & Moraceae & Streblus asper Lour. \\
\hline 99 & Rubiaceae & Canthium dicoccum (Gaertn.) Teijsm \& Binn. \\
\hline 100 & Rubiaceae & Morinda pubescens $\mathrm{Sm}$. \\
\hline 101 & Rubiaceae & Tricalysia apiocarpa (Dalz.) Gamble \\
\hline 102 & Rutaceae & Atalantia monophylla (L.) Corr.Ser \\
\hline 103 & Rutaceae & Chloroxylon swietenia DC. \\
\hline 104 & Rutaceae & Pamburus missionis (Wt.) Swingle \\
\hline 105 & Sapindaceae & Lepisanthes tetraphylla (Vahl) Radlk \\
\hline
\end{tabular}




\begin{tabular}{|c|c|c|}
\hline 106 & Sapotaceae & Madhuca longifolia (Koenig) J.Macbr. \\
\hline 107 & Sapotaceae & Mimusops elengi L. \\
\hline 108 & Sterculiaceae & Pterospermum canescens Roxb. \\
\hline 109 & Strychnaceae & Strychnos nux -vomica L. \\
\hline 110 & Verbenaceae & Premna tomentosa Willd. \\
\hline
\end{tabular}

Table 2. Phytosociological analysis of Shrubs in Konjikuppam sacred grove.

\begin{tabular}{|c|c|c|c|c|c|c|}
\hline Botanical Name & Indls. & RD & RF & $\mathbf{B A}$ & $\mathbf{R B A}$ & IVI \\
\hline Cadaba fruticosa & 16 & 13.94 & 0.20 & 0.048 & 0.11 & 0.45 \\
\hline Canthium coromandelianum & 12 & 3.35 & 3.46 & 8.010 & 1.37 & 9.13 \\
\hline Capparis divaricata & 6 & 16.62 & 11.63 & 0.64 & 1.44 & 40.68 \\
\hline Capparis rotundifolia & 8 & 0.40 & 10.20 & 0.01 & 0.44 & 2.26 \\
\hline Capparis sepiaria & 3 & 0.13 & 13.87 & 0.04 & 2.74 & 3.11 \\
\hline Catunaregam spinosa & 9 & 0.40 & 0.20 & 1.43 & 1.52 & 45.04 \\
\hline Clausena dentata & 35 & 8.57 & 0.37 & 4.18 & 0.41 & 14.02 \\
\hline Cormona retusa & 27 & 0.13 & 0.61 & 0.44 & 0.32 & 1.25 \\
\hline Dodonaea angustifolia & 12 & 0.40 & 4.48 & 2.79 & 0.44 & 12.07 \\
\hline Flacourtia indica & 6 & 3.75 & 3.67 & 0.37 & 0.74 & 14.02 \\
\hline Glycosmis mauritiana & 90 & 42.86 & 13.79 & 0.05 & 0.87 & 52.32 \\
\hline Grewia carpinifolia & 6 & 2.43 & 0.20 & 0.03 & 0.11 & 0.36 \\
\hline Grewia hirsuta & 4 & 0.80 & 1.22 & 0.11 & 0.46 & 1.16 \\
\hline Hugonia mystax & 21 & 3.36 & 4.69 & 1.52 & 0.21 & 22.28 \\
\hline Ixora pavetta & 5 & 2.58 & 0.20 & 0.65 & 0.27 & 1.11 \\
\hline Jatropha gossypifolia & 2 & 0.13 & 0.61 & 0.06 & 0.30 & 0.83 \\
\hline Memecylon umbellatum & 10 & 4.76 & 3.45 & 0.10 & 1.71 & 9.92 \\
\hline Mimosa intsia & 237 & 36.92 & 9.43 & 0.03 & 0.25 & 46.53 \\
\hline Ochna obtusata & 2 & 0.26 & 0.20 & 0.22 & 0.12 & 0.45 \\
\hline Opuntia dillenii & 5 & 0.40 & 0.40 & 0.05 & 0.02 & 0.38 \\
\hline Senna auriculata & 27 & 0.13 & 1.02 & 1.84 & 0.36 & 8.05 \\
\hline Tarenna asiatica & 11 & 0.27 & 0.20 & 0.97 & 0.41 & 3.11 \\
\hline Tragia plukeneti & 8 & 0.13 & 0.02 & 0.14 & 0.02 & 0.83 \\
\hline Zizyphus oenoplia & 3 & 0.26 & 3.46 & 0.75 & 0.64 & 1.37 \\
\hline
\end{tabular}


Table 3. Phytosociological analysis of Herbs in Konjikuppam sacred grove.

\begin{tabular}{|c|c|c|c|c|c|c|}
\hline Botanical Name & Indls. & RD & $\mathbf{R F}$ & BA & RBA & IVI \\
\hline Acanthospermum hispidum & 12 & 2.26 & 1.66 & 1.06 & 3.42 & 4.78 \\
\hline Achyranthes aspera & 25 & 1.02 & 0.96 & 0.48 & 2.67 & 2.12 \\
\hline Ageratum conyzoides & 17 & 1.53 & 1.39 & 0.72 & 2.77 & 16.65 \\
\hline Allmania nodiflora & 9 & 0.34 & 0.59 & 0.16 & 1.45 & 1.13 \\
\hline Alternanthera sessilis & 12 & 0.51 & 1.01 & 0.24 & 1.26 & 1.79 \\
\hline Alysicarpus monilifer & 13 & 0.45 & 0.21 & 0.21 & 5.25 & 0.69 \\
\hline Ammania baccifera & 8 & 0.60 & 0.85 & 0.28 & 1.75 & 1.51 \\
\hline Andrographis paniculata & 9 & 1.36 & 1.07 & 0.64 & 3.20 & 13.01 \\
\hline Asystasia gangetica & 14 & 0.26 & 0.32 & 0.12 & 2.00 & 0.85 \\
\hline Barleria cuspidate & 4 & 1.83 & 2.03 & 0.86 & 2.26 & 4.56 \\
\hline Barleria prionitis & 5 & 0.32 & 0.37 & 0.15 & 2.14 & 0.73 \\
\hline Blepharis molluginifolia & 8 & 0.60 & 0.43 & 0.28 & 3.50 & 1.10 \\
\hline Boerhaavia diffusa & 22 & 0.45 & 0.32 & 0.21 & 3.50 & 0.81 \\
\hline Cenchrus ciliaris & 40 & 0.51 & 0.69 & 0.24 & 1.85 & 1.24 \\
\hline Cereus trigonus & 3 & 2.26 & 2.08 & 1.06 & 2.72 & 7.25 \\
\hline Chloris barbata & 42 & 1.45 & 1.66 & 0.68 & 2.19 & 5.32 \\
\hline Chrysopogon aciculatus & 28 & 1.30 & 1.60 & 0.61 & 2.03 & 5.06 \\
\hline Cleome aspera & 12 & 2.58 & 2.78 & 1.21 & 2.33 & 8.98 \\
\hline Cleome monophylla & 9 & 0.38 & 0.37 & 0.18 & 2.57 & 0.99 \\
\hline Cleome viscosa & 18 & 2.88 & 2.19 & 1.35 & 3.29 & 5.26 \\
\hline Coldenia procumbens & 4 & 0.36 & 0.27 & 0.17 & 3.40 & 0.71 \\
\hline Commelina bengalensis & 16 & 0.45 & 0.59 & 0.21 & 1.91 & 1.78 \\
\hline Commelina erecta & 8 & 0.28 & 0.32 & 0.13 & 2.17 & 1.02 \\
\hline Corchorus aestuans & 11 & 0.26 & 0.27 & 0.12 & 2.40 & 0.88 \\
\hline Cynodon dactylon & 28 & 6.20 & 5.34 & 2.91 & 2.91 & 12.60 \\
\hline Dactyloctenium aegyptium & 19 & 0.47 & 0.69 & 0.22 & 1.69 & 1.28 \\
\hline Desmodium triflorum & 21 & 0.17 & 0.21 & 0.08 & 2.00 & 1.46 \\
\hline Dichanthium annulatum & 15 & 0.28 & 0.32 & 0.13 & 2.17 & 0.62 \\
\hline Diplachne fusca & 11 & 0.45 & 0.43 & 0.21 & 2.63 & 1.35 \\
\hline Ecbolium ligustrinum & 4 & 1.45 & 1.01 & 0.68 & 3.58 & 2.78 \\
\hline Eclipta prostrata & 29 & 1.79 & 2.19 & 0.84 & 2.05 & 4.06 \\
\hline Elytraria acaulis & 5 & 0.68 & 0.85 & 0.32 & 2.00 & 2.26 \\
\hline Heliotropium indicum & 3 & 0.60 & 0.59 & 0.28 & 2.55 & 1.21 \\
\hline Hybanthus enneaspermus & 8 & 0.38 & 0.32 & 0.18 & 3.00 & 0.73 \\
\hline Hygrophila auriculata & 13 & 2.69 & 2.99 & 1.26 & 2.25 & 6.39 \\
\hline Indigofera aspalathoides & 18 & 0.40 & 0.43 & 0.19 & 2.38 & 1.68 \\
\hline Lepidagathis cristata & 15 & 0.30 & 0.37 & 0.14 & 2.00 & 0.72 \\
\hline Leucas aspera & 56 & 1.04 & 1.60 & 0.49 & 1.63 & 3.65 \\
\hline Mollugo nudicaulis & 88 & 0.51 & 0.32 & 0.24 & 4.00 & 4.10 \\
\hline Ocimum canum & 22 & 0.26 & 0.21 & 0.12 & 3.00 & 0.74 \\
\hline Oldenlandia herbacea & 36 & 2.64 & 2.24 & 1.24 & 2.95 & 5.90 \\
\hline Oldenlandia umbellata & 43 & 0.34 & 0.43 & 0.16 & 2.00 & 1.13 \\
\hline Perotis indica & 13 & 1.83 & 1.12 & 0.86 & 4.10 & 3.19 \\
\hline Phyla nodiflora & 25 & 0.45 & 0.43 & 0.21 & 2.63 & 0.97 \\
\hline Physalis minima & 6 & 2.30 & 3.26 & 1.08 & 1.77 & 5.71 \\
\hline Scoparia dulcis & 9 & 1.68 & 1.55 & 0.79 & 2.72 & 4.68 \\
\hline Senna tora & 34 & 1.36 & 1.17 & 0.64 & 2.91 & 3.59 \\
\hline Sida cordifolia & 88 & 0.38 & 0.48 & 0.18 & 2.00 & 1.09 \\
\hline Solanum nigrum & 16 & 0.45 & 0.64 & 0.21 & 1.75 & 1.43 \\
\hline Sonchus oleraceus & 20 & 2.79 & 3.36 & 1.31 & 2.08 & 8.05 \\
\hline
\end{tabular}




\begin{tabular}{|c|c|c|c|c|c|c|}
\hline Sphaeranthus indicus & 15 & 6.84 & 5.34 & 3.21 & 3.21 & 13.34 \\
\hline Stachytarpheta jamaicensis & 56 & 6.35 & 5.34 & 2.98 & 2.98 & 13.06 \\
\hline Tribulus terrestris & 41 & 0.60 & 0.75 & 0.28 & 2.00 & 1.37 \\
\hline Triumfetta rhomboidea & 13 & 0.66 & 1.12 & 0.31 & 1.48 & 1.84 \\
\hline Vernonia cinerea & 27 & 0.28 & 0.21 & 0.13 & 3.25 & .58 \\
\hline Zornia triphylla & 6 & 1.79 & 1.66 & 0.84 & 2.71 & 3.83 \\
\hline
\end{tabular}

Table 4. Phytosociological analysis of Trees in Konjikuppam sacred grove.

\begin{tabular}{|c|c|c|c|c|c|c|c|}
\hline Botanical Name & Indls. & $\mathrm{RD}$ & $\mathrm{RF}$ & $\mathrm{BA}$ & RBA & IVI & BV \\
\hline Acacia auriculiformis & 2 & 0.67 & 1.10 & 0.50 & 1.27 & 3.04 & 0.45 \\
\hline $\begin{array}{c}\text { Acacia nilotica } \\
\text { ssp.indica }\end{array}$ & 2 & 0.67 & 2.20 & 0.95 & 2.39 & 5.26 & 1.23 \\
\hline Alangium salvifolium & 1 & 0.45 & 2.08 & 0.64 & 4.98 & 7.52 & 0.41 \\
\hline Albizia amara & 43 & 20.48 & 10.34 & 0.71 & 12.62 & 43.44 & 0.73 \\
\hline Albizia lebbeck & 2 & 0.67 & 2.20 & 0.95 & 2.39 & 5.26 & 1.23 \\
\hline Atalantia monophylla & 3 & 1.43 & 3.45 & 0.50 & 8.95 & 13.82 & 0.19 \\
\hline Azadirachta indica & 1 & 0.48 & 3.45 & 0.20 & 3.50 & 7.42 & 0.08 \\
\hline Canthium dicoccum & 4 & 1.90 & 10.34 & 0.03 & 0.56 & 12.81 & 0.01 \\
\hline Cassia fistula & 6 & 2.29 & 8.33 & 0.29 & 2.22 & 13.24 & 0.07 \\
\hline Cassine glauca & 3 & 1.35 & 2.08 & 0.03 & 0.25 & 3.67 & 0.23 \\
\hline Chloroxylon swietenia & 12 & 5.71 & 10.34 & 0.71 & 12.62 & 28.68 & 0.73 \\
\hline Cordia monoica & 2 & 1.83 & 2.27 & 3.14 & 18.01 & 22.12 & 2.83 \\
\hline Cordia obliqua & 8 & 2.69 & 3.30 & 0.79 & 1.98 & 7.97 & 1.52 \\
\hline Dichrostachys cinerea & 1 & 0.93 & 2.27 & 0.03 & 0.18 & 3.33 & 0.01 \\
\hline Diospyros melonoxylon & 6 & 3.37 & 2.20 & 0.13 & 0.38 & 5.88 & 0.11 \\
\hline Diospyros montana & 4 & 1.79 & 4.17 & 0.36 & 2.85 & 8.81 & 0.23 \\
\hline Ficus amplissima & 1 & 0.92 & 2.27 & 0.10 & 0.55 & 3.74 & 0.02 \\
\hline Ficus racemosa & 1 & 0.34 & 0.10 & 0.13 & 0.32 & 1.75 & 0.15 \\
\hline Ficus religiosa & 1 & 0.45 & 2.04 & 1.52 & 12.06 & 14.59 & 1.39 \\
\hline Lannea coromandelica & 1 & 0.48 & 3.45 & 0.71 & 12.62 & 16.54 & 0.73 \\
\hline Lepisanthes tetraphylla & 25 & 22.94 & 11.36 & 2.99 & 17.12 & 51.42 & 3.85 \\
\hline Madhuca longifolia & 14 & 6.28 & 4.17 & 0.50 & 3.94 & 14.38 & 0.32 \\
\hline Mimusops elengi & 4 & 3.67 & 6.82 & 1.13 & 6.48 & 16.97 & 2.19 \\
\hline Morinda pubescens & 7 & 3.14 & 8.33 & 0.05 & 0.38 & 11.56 & 0.01 \\
\hline Pamburus missionis & 10 & 3.37 & 2.20 & 0.20 & 0.51 & 6.08 & 0.26 \\
\hline Premna tomentosa & 4 & 1.79 & 4.17 & 0.03 & 0.25 & 6.21 & 0.01 \\
\hline $\begin{array}{l}\text { Pterospermum } \\
\text { canescens }\end{array}$ & 2 & 0.95 & 3.45 & 0.05 & 0.87 & 5.27 & 0.01 \\
\hline Streblus asper & 5 & 4.59 & 2.27 & 0.50 & 2.88 & 9.74 & 0.45 \\
\hline Strychnos nux-vomica & 1 & 0.45 & 2.08 & 0.88 & 6.91 & 9.45 & 1.37 \\
\hline Tricalysia apiocarpa & 21 & 1.83 & 2.27 & 0.04 & 0.22 & 44.33 & 0.02 \\
\hline Walsura trifoliata & 1. & 0.45 & 2.08 & 1.52 & 11.89 & 14.42 & 2.35 \\
\hline
\end{tabular}


Table 5. Consoldidate Details of Phytosocialogical Analysis of the Sacred Groves in Konjikuppam Village Cuddalore District.

\begin{tabular}{|c|c|c|c|c|}
\hline $\begin{array}{l}\text { Sl. } \\
\text { No. }\end{array}$ & Criteria & $\begin{array}{l}\text { Disturbed } \\
\text { Site I }\end{array}$ & $\begin{array}{c}\text { Moderately } \\
\text { Disturbed } \\
\text { Site II }\end{array}$ & $\begin{array}{l}\text { Undisturbed } \\
\text { Site III }\end{array}$ \\
\hline 1. & \multicolumn{4}{|c|}{ Number of species } \\
\hline & Trees & 9 & 17 & 26 \\
\hline & Saplings & 2 & 7 & 11 \\
\hline & Seedlings & 3 & 6 & 22 \\
\hline & Shrubs & 6 & 13 & 18 \\
\hline & Herbs & 12 & 29 & 43 \\
\hline 2. & \multicolumn{4}{|c|}{ Density(no/ha) } \\
\hline & Trees & 19 & 57 & 83 \\
\hline & Saplings & 120 & 190 & 160 \\
\hline & Seedlings & 630 & 710 & 830 \\
\hline & Shrubs & 120 & 420 & 313 \\
\hline & Herbs & 4250 & 9200 & 11280 \\
\hline 3. & \multicolumn{4}{|c|}{ Basal area(m2/ha) } \\
\hline & Trees & 4.565 & 8.383 & 13.311 \\
\hline & Saplings & 2.356 & 4.515 & 8.050 \\
\hline & Seedlings & 0.301 & 0.458 & 0.519 \\
\hline & Shrubs & 1.057 & 2.213 & 2.057 \\
\hline & Herbs & 0.063 & 0.087 & 0.272 \\
\hline 4. & \multicolumn{4}{|c|}{ Diversity index } \\
\hline & Trees & 1.103 & 1.572 & 2.054 \\
\hline & Saplings & 0.958 & 1.856 & 2.169 \\
\hline & Seedlings & 1.048 & 1.571 & 2.209 \\
\hline & Shrubs & 1.247 & 2.053 & 2.307 \\
\hline & Herbs & 1.637 & 2.002 & 2.056 \\
\hline 5. & \multicolumn{4}{|c|}{ Dominance index } \\
\hline & Trees & 0.267 & 0.150 & 0.125 \\
\hline & Saplings & 0.430 & 0.168 & 0.104 \\
\hline & Seedlings & 0.324 & 0.164 & 0.096 \\
\hline & Shrubs & 0.159 & 0.120 & 0.095 \\
\hline & Herbs & 0.194 & 0.135 & 0.127 \\
\hline 6. & \multicolumn{4}{|c|}{ Species richness } \\
\hline & Trees & 0.863 & 1.042 & 1.566 \\
\hline & Saplings & 0.500 & 1.299 & 2.157 \\
\hline & Seedlings & 0.378 & 0.548 & 1.185 \\
\hline & Shrubs & 1.223 & 0.976 & 1.336 \\
\hline & Herbs & 1.211 & 0.913 & 1.175 \\
\hline 7. & \multicolumn{4}{|c|}{ Evenness index } \\
\hline & Trees & 2.154 & 2.073 & 2.097 \\
\hline & Saplings & 2.007 & 2.183 & 2.128 \\
\hline & Seedlings & 2.021 & 2.247 & 2.132 \\
\hline & Shrubs & 2.081 & 2.239 & 2.250 \\
\hline & Herbs & 1.839 & 2.158 & 2.880 \\
\hline
\end{tabular}


Table 6. Particulars regarding the Ethnomedicinal plants in the sacred grove, Konjikuppam, Cuddalore District, Tamil Nadu.

\begin{tabular}{|c|c|c|c|c|}
\hline S.No & Botanical name & $\begin{array}{l}\text { Diseases/ } \\
\text { Disorders }\end{array}$ & $\begin{array}{l}\text { Parts } \\
\text { used }\end{array}$ & Mode of application \\
\hline 1. & $\begin{array}{l}\text { Albizia lebbeck (L.) } \\
\text { Benth. }\end{array}$ & Snake bite & Flower & $\begin{array}{c}\text { The powder prepared from } 5 \mathrm{gm} \text { of flower is taken } \\
\text { orally along with hot water three times a day for } \\
\text { three days }\end{array}$ \\
\hline 2. & Aristolochia indica $\mathbf{L}$. & Snake bite & Root & $\begin{array}{l}\text { Dried roots are powdered and about } 10 \mathrm{~g} \text { powder is } \\
\text { given with } 100 \mathrm{ml} \text { of human urine as an antidote for } \\
\text { snake bite. }\end{array}$ \\
\hline 3. & $\begin{array}{l}\text { Asystasia gangetica } \\
\text { (L.) T.Anderson }\end{array}$ & Wounds & Leaves & $\begin{array}{l}\text { Leaf powder is mixed with coconut oil and applied } \\
\text { topically to heal wounds (burns). }\end{array}$ \\
\hline 4. & Barleria prionitis $\mathbf{L}$. & $\begin{array}{l}\text { whooping } \\
\text { cough }\end{array}$ & $\begin{array}{l}\text { Whole } \\
\text { plant }\end{array}$ & $\begin{array}{l}\text { About } 20 \mathrm{ml} \text { of plant juice is used for 3-4 days twice } \\
\text { daily, with a pinch of salt to treat whooping cough }\end{array}$ \\
\hline 5. & $\begin{array}{l}\text { Blepharis } \\
\text { molluginifolia Pers. }\end{array}$ & $\begin{array}{l}\text { Bone } \\
\text { fracture }\end{array}$ & Leaves & $\begin{array}{l}\text { Leaf paste is mixed with the powdered black gram, } \\
\text { crushed onion and white yolk of one egg and the } \\
\text { mixture is applied topically over the fractured } \\
\text { bones. }\end{array}$ \\
\hline 6. & Capparis brevispina $\mathrm{L}$. & Migraine & Shoot & $\begin{array}{c}\text { Decoction of young branches is taken to cure } \\
\text { migraine. }\end{array}$ \\
\hline \multirow{2}{*}{$\begin{array}{l}7 . \\
8 .\end{array}$} & \multirow[t]{2}{*}{ Capparis zeylanica $\mathrm{L}$. } & Syphilis & Roots & $\begin{array}{l}\text { Roots are ground together with } 21 \text { black peppers } \\
\text { and the powder is taken with watere daily for } \\
\text { getting relief in syphilis }\end{array}$ \\
\hline & & $\begin{array}{c}\text { Leucorrhea } \\
\text { Menorrhoea }\end{array}$ & $\begin{array}{l}\text { Leaf, } \\
\text { seed }\end{array}$ & $\begin{array}{l}\text { Leaves and seeds are ground into paste and made } \\
\text { into pills. Three pills are given orally with lime } \\
\text { water for } 3 \text { days. }\end{array}$ \\
\hline 9. & Cissampelos pariera $\mathrm{L}$. & $\begin{array}{l}\text { Fever, } \\
\text { Headache }\end{array}$ & Shoot & $\begin{array}{l}\text { 5-10 } \mathrm{ml} \text { juice of aerial part is taken orally twice a } \\
\text { day before meals. }\end{array}$ \\
\hline 10. & $\begin{array}{l}\text { Clausena dentata } \\
\text { (Willd.) M.Roem }\end{array}$ & Wounds & Leaves & Paste of leaves is applied over the affected area. \\
\hline 11. & Cleome viscosa $\mathrm{L}$. & Ear ache & Root & $\begin{array}{l}\text { Root is pounded with garlic, mixed with castor oil, } \\
\text { boiled and juice is squeezed; about } 3 \text { drops applied } \\
\text { in the ear to treat ear ache. }\end{array}$ \\
\hline 12. & $\begin{array}{l}\text { Chloroxylon swietenia } \\
\text { DC. }\end{array}$ & Diabetes & Gum & $\begin{array}{l}\text { About } 100 \mathrm{ml} \text { gum powder mixed with water is } \\
\text { given for about one month for the treatment of } \\
\text { diabetes. }\end{array}$ \\
\hline 13. & $\begin{array}{l}\text { Coccinia grandis } \\
\text { (L.)Voigt }\end{array}$ & $\begin{array}{l}\text { Excess } \\
\text { bleeding }\end{array}$ & $\begin{array}{l}\text { Whole } \\
\text { plant }\end{array}$ & $\begin{array}{l}\text { 2-3 tablespoons of fresh plant juice is recommended } \\
\text { for 2-3 days to check bleeding during pregnancy }\end{array}$ \\
\hline 14. & $\begin{array}{l}\text { Coldenia procumbens } \\
\text { L. }\end{array}$ & $\begin{array}{l}\text { White } \\
\text { discharge }\end{array}$ & Leaves & $\begin{array}{l}\text { Juice of leaf is taken orally to prevent white } \\
\text { discharge in women. }\end{array}$ \\
\hline 15. & $\begin{array}{l}\text { Diospyros montana } \\
\text { Roxb. }\end{array}$ & Diabetes & Leaves & $\begin{array}{l}\text { 2-3 teaspoonfuls extract of fresh stem bark taken in } \\
\text { stomachache. }\end{array}$ \\
\hline 16. & Ficus racemosa $\mathrm{L}$. & $\begin{array}{l}\text { Diarrhea and } \\
\text { Dysentery }\end{array}$ & Leaves & $\begin{array}{l}\text { About } 5 \text { teaspoonful of decoction of stem bark is } \\
\text { given twice a day for about } 2 \text { days to treat diarrhea } \\
\text { and dysentery }\end{array}$ \\
\hline 17. & $\begin{array}{l}\text { Hygrophila auriculata } \\
\text { (Schum) Heine }\end{array}$ & Jaundice & $\begin{array}{l}\text { Leaves, } \\
\text { seeds }\end{array}$ & $\begin{array}{l}\text { Leaves and seeds are ground into paste and made } \\
\text { into pills, } 2 \text { pills per day taken for } 5 \text { days. Diet: } \\
\text { Buttermilk and food, salt restricted. }\end{array}$ \\
\hline 18. & $\begin{array}{l}\text { Madhuca longifolia } \\
\text { (Koenig) J.Macbr. }\end{array}$ & Asthma & $\begin{array}{c}\text { Flower } \\
\text { s }\end{array}$ & $\begin{array}{l}\text { Five flowers are boiled in a glass of water until it is } \\
\text { reduced to half and is administered orally once a } \\
\text { day }\end{array}$ \\
\hline 19. & Mimusops elengi L. & Fever & Bark & $\begin{array}{l}\text { Bark powder mixed with red sugar in applied on } \\
\text { jaw in toothache }\end{array}$ \\
\hline
\end{tabular}




\begin{tabular}{|c|c|c|c|c|}
\hline 20. & $\begin{array}{l}\text { Mukia maderapatana } \\
\text { (L.) M.Roem }\end{array}$ & $\begin{array}{l}\text { Cold and } \\
\text { cough }\end{array}$ & Leaves & $\begin{array}{c}\text { Leaf powder is mixed with boiled rice and taken } \\
\text { orally to treat cold and cough }\end{array}$ \\
\hline 21. & $\begin{array}{l}\text { Phyla nodiflora (L.) } \\
\text { Greene }\end{array}$ & Tooth ache & Leaves & $\begin{array}{l}\text { Leaves ground with Allium sativum and made into } \\
\text { pills kept between teeth to check toothache }\end{array}$ \\
\hline 22. & $\begin{array}{l}\text { Sphaeranthus indicus } \\
\text { Linn. }\end{array}$ & $\begin{array}{l}\text { Blood } \\
\text { dysentery }\end{array}$ & Root & $\begin{array}{l}\text { Dried powdered root with } 5 \text { black pepper (Piper } \\
\text { nigrum). Powdered drug given } 3 g \text { per dose twice a } \\
\text { day }\end{array}$ \\
\hline 23. & $\begin{array}{c}\text { Stachytarpheta } \\
\text { jamaicensis (L.) Vahl } \\
\end{array}$ & Dysentery & $\begin{array}{l}\text { Stem, } \\
\text { Root }\end{array}$ & $\begin{array}{c}\begin{array}{c}\text { Paste of stem and root bark is applied topically to } \\
\text { treat dysentery }\end{array} \\
\end{array}$ \\
\hline 24. & $\begin{array}{l}\text { Tiliacora acuminata } \\
\text { (Lamk.) Miers ex } \\
\text { Hook.f.\& Thoms. }\end{array}$ & $\begin{array}{c}\text { Constipatio } \\
\mathbf{n}\end{array}$ & Stem & $\begin{array}{l}\text { About } 100 \mathrm{~g} \text { pieces of stem are powdered and one } \\
\text { teaspoonful is given with warm water twice a day } \\
\text { after meals for two weeks to treat constipation }\end{array}$ \\
\hline
\end{tabular}

\section{CONCLUSIONS}

There are about 24 species used by local communities of Konjikuppam recorded by using structured questionnaire. Although the same species used to treat the same ailment in various parts of Tamilnadu but the mode of preparation and administration is vary. (Table.6) during each field survey, they consented orally to document and publish the results for the study of society. After initial reconnaissance survey of the area in and discussions with the local people, a total of 15 resource persons, comprising of 10 males and 5 females were identified. These are locally referred to as vaidyas and perform the duties of medicinal practitioner. Information on the habitat of the plant, local name of plant, plant part used for curing, method of dosage and administration were recorded.

The Konjikuppam ( 4.5 ha species) representing 96 genera and 48 families there were 32 trees species, 24 shrubs, 56 hers. The konji plant Glycomis mauritiana was the dominant species followed by Albizia amara and Tricalysia sphaerocarpa.the Shannon index was 1.72 and eveness of 0.65 heavy undergrowth separately interrupted by trees characterizes the vegetation. The study has confirmed the existence of ecological and medical traditions and documented untapped, useful and valuable information from a tiny tot on the Coromandel ocast of India dominated by a communties. A treasure trove of knowledge awaits the feature researchers as there are 4635 ethnic communities spread over India landscape.

On the otherhand, eventhough the plants somewhat disappeared and also affected due to the Thanae cyclone hitted especially in Cuddalore district on December 30. Due to the devastation of species diversity in the study area there is an urgent need for regeneration of the species for conservation of species and their biodiversity.

\section{References}

[1] Bhandary, M.J. and K.R Chandrasekar. 2003. Sacred groves of Dakhina Kanada and Udupi districts of Karnataka. Curr.Sci. 85:1655-1656.

[2] Curtis, J.T. 1959. The vegetation of Wisconsin. An ordination of plant communities, MadisonWinconsin.

[3] Gadgil, M. and Vartak, V.D. 1975. Sacred groves of India: a plea for continued conservation. J. Bombay Nat. His. Soc., 72: 314-320.

[4] Haridasan .K. and P.R. Rao. 1985. Forest Flora of Mehalaya, Vol.1, Bishen Singh and Mhendrapal Singh, Dhera Dun, India. 
[5] Haridasan Nair., G. Gopikumar, K., Pramod, G., Krishnan and Sunil kumar K.K. 1997. Sacred groves of India vanishing greenery. Current Science. 72: (10) 697- 699.

[6] Jain, S.K, 1989. Methods and Approaches in Ethnobotany, Society of Ethnobotanist, Lukhnow.

[7] Khan,M. K., S. Mohan and K.S. Bawa. 1997. Effectiveness of The Protected Area Network in Biodiversity Conservation : a case study of Mehalaya State. Biodiv. Conserve. 6:853-868.

[8] Margalef, R. 1958. Perspectives in Ecological theory. Univ. of Chicago Press,pp.111.

[9] Martin, G. 1995. Ethnobotany - A Method Manual. Chapman and Hall, London.

[10] Menhinck, E.F. 1964. A comparison of some species diversity indices applied to samples of field insects. Ecology, 45:859-861.

[11] Nadanakunjidam, S. and G. Kamashi. 2003. Traditional medicinal knowledge of a few plants of Pondicherry and Karaikal region, Pondicherry. Adv. Plant Sci. 16:405-412.

[12] Nadanakunjidam, S.and S.Abirmi. 2005. Comparative study of traditional medicinal knowledge of Pondicherry and Karaikal region in Union Territory of Pondicherry. Ethnobotany 17: 112117.

[13] Nadanakunjidam,S. 2006. Some intresting medicament from traditional medicinal practioners of Karaikal region, Pondicherry. J. Eco.Taxon. Bot. 30: 449-452.

[14] Odum, E.P.1971. Fundamentals of Ecology. $3^{\text {rd }}$ edn. W.B.Saunders Co. Philadelphia.

[15] Osambi, D.D. 1962. Myth and Reality, popular press, Bombey,Iindia.

[16] Peet, R.K. 1974. Patterns of species diversity. Ann Rev. Ecol. Syst.,285-307.

[17] Pielou, E.C.1966. The measurement of diversity in different types of biological collections. J.Theor.Biol.,13:131-144

[18] Ramanujam M.P. and. D. Kadamban. 2001. Plant biodiversity of two tropical dry evergreen forest in the Pondicherry region of south India and the role of belief system in their conservation. Biodiv. Conserve. 10: 1203-1217.

[19] Ramanujam, M.P. and K. Pravin kumar Cyril. 2003. Woody species diversity of four sacred groves in the Pondicherry region of South India. Biodiv. Conserve. 12: 289- 299.

[20] Simpson, E.H.1949. Measurement of Biodiversity. Nature (London) 163:688.

[21] Vertak V.D. and Gadgil, Dev Rahati. 1973. An ethnobotanical study of the forest preserved on grounds of religious belief. Proc. Indian Sci. Cong. 60: 341. 